\title{
SOIAR IRRADIANCE VARIATIONS AND THEIR RELATION WITH SOLAR FLARES
}

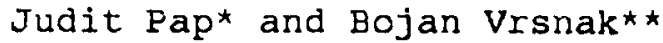

*Kiepenheuer-Institut fuer Sonnenphysik

D-7800 Freiburg, Schoeneckstr. 6, F.R.G.

**Hvar Observatory of Zagreb University,

Y-41000 Zagreb, Kaciceva 26, Yugoslavia.

A clear association is demonstrated between the dips in the total solar irradiance and flare occurrence. It is found that both the irradiance dips and flares are related to emerging new activity.

\section{INTRODUCTION}

The temporary irradiance dips are the most obvious events in the total solar irradiance variation indicated by the SMM/ACRIM radiometer $/ 1 /$. It has been shown that sunspots reduce the total irradiance $/ 1,2 /$ and the evolution of the active regions also plays an important role in the short-term irradiance variations $/ 3,4 /$. It has also been found that solar flares are related to the emergence of magnetic fields on the surface $/ 5,6 /$.

The purpose of this paper is to investigate the relation between the irradiance dips, and solar flares. The irradiance measurements of the SMM/ACRIM radiometer are used for 1980. The daily means of the projected sunspot areas are taken from the Solnechnye Dannye Bulletin. The age irformation on active regions is taken from the Solar Geophysical Data (SGD) catalogue, after identification of the active regions published in the two catalogues. The daily numbers of flares and coronal mass ejections (CME) are determined from $S G D$.

\section{IRRADIANCE VARIATION AND FLARE OCCURRENCE}

Figere 1 shows the correlation between the SMM/ACRIM data, projected sunspot areas and solar flares. Active sunspot groups are defined as young and quickly developing complex groups /4/. The passive ones are the simple old groups. As can be seen during the irradiance dips, when active spots have large areas, the number of flares of complex active regions is also large. No significant correlation is found between the irradiance dips and old active regions, including their simple spots and flares. The number of the coronal mass ejection is also enhanced around the irradiance dips but they are not necessarily connected to them.

The correlation coefficients between tie ACRIM irradiance data and spot areas and flare indices as well as CME are given in Table 1. As it is expected, the correlation is strong between the

TABLE 1

Correlation coefficients between the ACRIM data and various activity indices are given for the time interval 1980 March-Nov.

\begin{tabular}{|c|c|c|c|c|c|c|}
\hline & $\begin{array}{c}\operatorname{daily} \\
\text { all }\end{array}$ & $\begin{array}{l}\text { flare no. } \\
\text { complex }\end{array}$ & $\begin{array}{l}\text { in } A R^{\prime} s \\
\text { old }\end{array}$ & CME & $\begin{array}{l}\text { projected } \\
\text { active }\end{array}$ & $\begin{array}{c}\text { spot areas } \\
\text { passive }\end{array}$ \\
\hline $\begin{array}{l}\text { ACRIM } \\
\text { active spots } \\
\text { passive spots }\end{array}$ & $\begin{array}{r}-0.46 \\
0.54 \\
0.08\end{array}$ & $\begin{array}{r}-0.57 \\
0.60 \\
-0.07\end{array}$ & $\begin{array}{r}0.21 \\
-0.11 \\
0.37\end{array}$ & $\begin{array}{r}-0.18 \\
0.20 \\
-0.08\end{array}$ & $\begin{array}{r}-0.84 \\
-.-1\end{array}$ & 0.30 \\
\hline
\end{tabular}


irradiance data and active spot areas with a correlation coefficient of -0.84 , that is -0.57 for the daily flare number of complex active regions. No high correlation coefficients are found for the old spots, flares in old active regions, and CME.

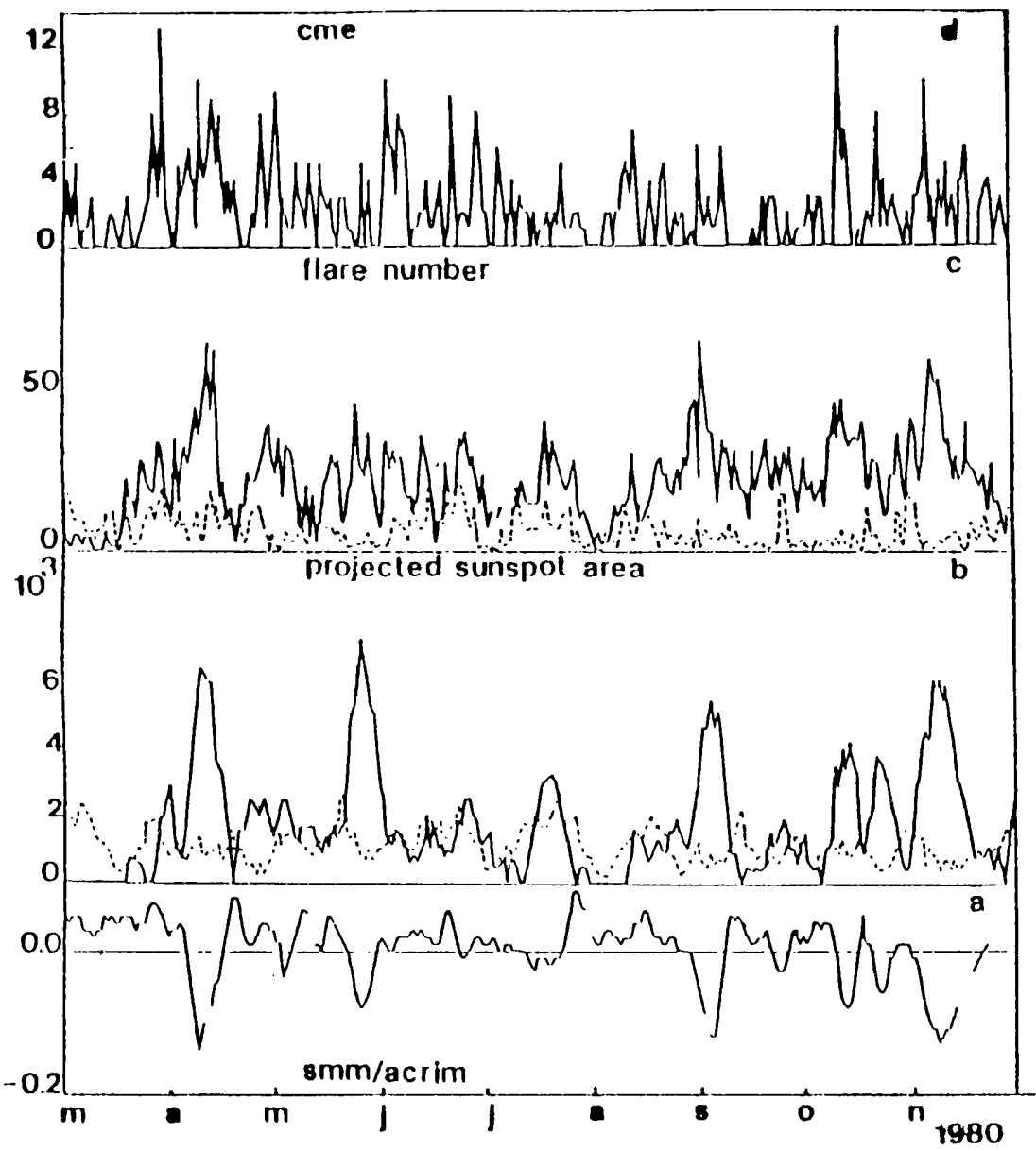

Fig. 1. (a) shows the SMM/ACRIM data, full lines of (b) and (c) show the projected areas of active spots and daily number of flares occuring in young complex active regions, respectively. Dashed lines of (b) and (c) show the spot areas and daily flare numbers in old active regions, respectively. (d) gives the daily numbers of the CME's. The curves refer to the time interval March-November, 1980.

\section{ENERGETICS OF SOLAR FLARES}

The increased flare activity in the active regions which produce dips in the total solar irradiance indicates an enhanced deposition of free magnetic energy into the coronal magnetic structures (so-called flare built up process). The storage of energy can be a result of local dynamo driven by convective motions, or, as our statistics also demonstrates, can be a consequence of the emerging flux process.

In the first case, when the magnetic field lines are twisted by the rotating plasma motions on the solar surface, the energy flux transported into the corona can be expressed as:

$$
F=v_{\varphi} B_{\varphi} B_{Z} / \mu_{0}
$$

$v \varphi$ is the rotational velcsity, $\mathrm{B} \varphi$ and $\mathrm{B} z$ are the axial and azimuthal components of the magnetic field. Taking $B_{z}=10^{-1} \mathrm{~T}$ for 
a sunspot magnetic field and using $B_{\varphi}=10^{-2} \mathrm{~T}$ and $\mathrm{v}_{\varphi}=100 \mathrm{~m} / \mathrm{s}$, the $F$ energy flux transported to the corona is in the order of $10^{5}$ $\mathrm{W} / \mathrm{m}^{2}$. In the case of strong, twisted fields the energy flux transported to the corona should be up to $10^{6} \mathrm{~W} / \mathrm{m}^{2}$.

The energy which is carried up by emerging flux can be calculated as

$$
F=v_{e} B^{2} / 2 \mu_{0}
$$

where $B$ magnetic field is emerging with $v$ velocity. Using $B=10^{-1}$ $T$ and $v e=100 \mathrm{~m} / \mathrm{s}$, the energy transported to the corona is again in the order of $10^{5} \mathrm{~W} / \mathrm{m}^{2}$. Thus, if we take into account a sunspot with an area of 1000 ppm of the hemisphere, the order of the total energy, which can be transported and stored in the coronal magnetic fields, is $10^{20}-10^{24} \mathrm{~W}$.

We can directly estimate the energy released by flares from the daily scaled flare number (SFN). The scaling is performed by means of the soft X-ray measurements of the GOES satellite in the range 1-8 A /7/. The SFN is defined as:

$$
\mathrm{SFN}=\sum_{i m p} \mathrm{~K}_{l m p} \mathrm{~N}_{l m p}
$$

where $K_{\text {limpis }}$ the scaling coefficient and $N_{\text {lmp }}$ is the daily number of flares in a particular class of importances. Using a statistical sample of flares of different importances, average values of soft $X$-ray peak fluxes have been determined for particular classes of flare importance. A roughly exponential dependence has been obtained and the best fit gives the $K_{\text {lmp }}$ scaling coefficients listed in Table 2 .

TABLE 2

\begin{tabular}{|c|c|c|c|c|c|c|c|}
\hline importance & $S f, S n$ & $\mathrm{Sb}, 1 \mathrm{n}, 1 \mathrm{f}$ & $1 \mathrm{~b}$ & $2 f, 2 n$ & $2 b$ & $3 f, 3 n$ & $3 b$ \\
\hline $\mathrm{K}_{i m p}$ & 0.5 & 1 & 2 & 5 & 10 & 20 & 50 \\
\hline
\end{tabular}

Values of $K_{\lambda m p}$ scaling coeffiecients are given after $/ 7 /$.

We assume that the total energy liberated by flares of different importances is scaled in the same way as the average soft $X-r a y$ in the order of $10^{25} \mathrm{~J} / 8 /$, the daily energy released by flares can be expressed as:

$$
E=S F N \star 10^{25} / 50
$$

It is found that SFN is between 30 and 50 , which gives an energy released by flares in the order of $10^{25} \mathrm{~J} /$ day, corresponding to about $10^{20} \mathrm{w}$.

Beside the flares, the energy which is carried by large MHD blast can be up to $10^{25} \mathrm{~J} / 8 /$. Thus, an upper limit of energy carried by CME's can be calculated as:

$$
E_{C M E}<N_{C M E} * 10^{25}
$$

Since during the irradiance dips NCME is up to $10 /$ day $/ 7 /$, the upper limit of the energy transported by CME's is smaller than $1026 \mathrm{~J} /$ day $(1021 \mathrm{~W})$.

Recently, Lawrence / $9 /$ has calculated the order of an irradiance 
decrease $(\triangle S)$ for a sunspot group with area of $1000 \mathrm{ppm}$ of the hemisphere. The value of $\Delta S$, according to his calculation, is $6 * 10^{22} \mathrm{~W}$. Similarly, taking into account a "mean" irradiance dip with an amplitude $1 \mathrm{~W} / \mathrm{m}^{2}(0.05 \%)$ and with a 10 days duration and assuming a time-dependent, axially symmetric perturbation of the irradiance by integrating the perturbation over the solid angle $2 \pi$, we can obtain $\Delta S=3 \times 10^{22} \mathrm{w}$. That is, the energy which might be carried out by flares and CME's is at least one order less than the missing energy in a mean irradiance dip, as it was also calculated by $/ 10 /$.

\section{CONCLUSIONS}

Our results demonstrate a strong correlation between the SMM/ACRIM irradiance data, active sunspot areas and solar flares. It is found that between March and November 1980 most of the solar flares occurred in young complex active regions which caused the dips in the total solar irradiance.

It has been suggested /11/ that the up-drafting boundaries of giant convective cells carry magnetic fields from the interior of the sun and become the seats of active regions. Occurrence of flares, formation of sunspots or the new activity of older ones and dips in the total solar irradiance can be regarded as manifestations of emergence of magnetic fields on the solar surface.

The increased flare" activity in the active regions which play a role in producing irradiance dips indicates an enhanced deposition of free magnetic energy into the coronal magnetic structures (flare built up process). Although, the energy carried by flares and CME's is small, at least one order of magnitude smaller than the missing energy of the irradiance dips, these investigations might help to understand better the physical origin of the irradiance variations and related events.

Acknowledgements: The authors would like to express their gratitude to Dr. R.C. Willson for providing the SMM/ACRIM data. One of us (J.P.) expresses her gratitude for Prof. E. Schroeter for allowing this research at the Kiepenheuer-Institut and for the Deutsche Forshungsgemeinschaft (Bonn) for the financial support. We also would like to thank to Prof. M. Stix the critical reading of the manuscript; and to H.P. Schilling (Kiepenheuer-Inst.) and H.Bozis (Hvar Obs.) for their assistance.

\section{REFERENCES}

1. Willson, R.C., Gulkis, S., Jansenn, M., Hudson, H.S., and Chapinan, G.A.: 1981 , Science 211, 700.

2. Hudson, H.S., Silva, S., Woodard, M., and Willson, R.C.: 1982 , Solar Physics 76, 211.

3. Willson, R.C.: 1982, Journal of Geophysical Research 87,4319.

4. Pap, J.: 1985, Solar Physics 97, 21.

5. Zirin, H.: 1970 , Solar Physics 14, 328.

6. Ichimoto, K., Kubota, J., Suzuki, M., Tohmura, I., and Kurakova, H.: 1985, Nature $316,422$.

7. Vrsnak, B., and Ruzdjak, V.: 1988 , Adv. Space Res., in press.

8. Priest, E.: 1982, in Solar Magnetohydrodynamics, D. Reidel Publ. Company.

9. Lawrence, J.K.: 1987, Journal of Geophysical Research 92D, 796.

10. Hudson, H.S., and Wilison, R.C.: 1983, Solar Physics $86,123$.

11. Bai, T.: 1987, The Astrophysical Journal 314, 795. 